\title{
Small Molecule Inhibitors and Inflammatory Skin Diseases
}

\author{
Hanan H. Omar \\ Clinical Pathology Department, Faculty of Medicine, Suez Canal University, Egypt
}

\begin{abstract}
Inflammatory skin diseases (ISDs) are originally a consequence of many processes of protective and regenerative skin responses against infections and dangers. A characteristic feature of each ISD is the production of disease-relevant cytokines by local immune and epithelial cells. The JAKSTAT pathway is necessary for a wide range of cytokines and growth factors, leading to critical cellular events. Cytokines that signal through type I/II cytokine receptors typically activate at least one JAK family member and one or more STAT proteins. Many of immunological disorders have been happened through different cytokine receptors throughout the JAK-STAT pathway, particularly $\mathrm{T}$ cell mediated diseases. Thus, targeting of this pathway has gained huge attraction. New drugs were introduced to inhibit JAK and STAT molecules. Although only few JAK inhibitors (JAKinib) are FDA approved, other JAKinibs and possible STAT inhibitors are being developed by passing preclinical evaluations and clinical trials. For example, the oral JAK1/JAK2 inhibitors ruxolitinib and baricitinib both seem to induce hair-regrowth in patients with Alopecia areata. The tofacitinib, (JAK1/JAK3 inhibitor) showed an improvement of $>75 \%$ of the psoriasis area. the first generation which targeting multiple JAK/STAT-associated cytokines at the same time as the second generation which targeting the signaling pathway itself is an alternative approach to neutralizing single cytokines by antibodies.
\end{abstract}

Keywords: inhibitor, JAKi, STAT

\section{Introduction}

Inflammatory skin diseases are originally a consequence of many processes of protective and regenerative skin responses against infections and dangers. The dermatitis is occurred due to hereditary or acquired disorder in a specific layer of the host defense system when the causative infections and dangers are ruled out ${ }^{(1)}$. Many cytokines present during skin inflammation, such as IL-4, IL-22, IL-23, and IFNs, signal through type I/II cytokine receptors.
These receptors lack intrinsic kinase activity and base on a family of associated cytoplasmic protein kinases upon stimulation to signal within the cell(2). The Janus kinasesignal transducer and activator of transcription (JAK-STAT) pathway plays a most important role in transferring of signals from cell-membrane receptors to the nucleus (3). JAK-STAT pathway is necessary for many cytokines and growth factors, leading to critical cellular events, i.e. hematopoiesis, lactation and development of the immune system and mammary glands ${ }^{(4)}$. 


\section{JAK-STAT Pathway}

STATs were firstly found in 1988 as proteins that combine with interferon (IFN)stimulated response elements of DNA sequences in order to stimulate the transcription of type I IFNs. Then, JAKs were discovered in 1992 by three separate labs and the JAK-STAT pathway was coined. The name of the JAK comes from a Roman two-faced god that implies two domains, including a catalytic domain and a kinase-like domain. Type I- and II receptors are associated with $\mathrm{JAKs}^{(5)}$. The binding of ligand (cytokine) to its receptor results in receptor dimerization and subsequently, JAKs are activated following close proximity. These activated JAKs initiate trans-phosphorylation on specific tyrosine residues, generating docking sites for recruitment of latent cytoplasmic transcription factors ${ }^{(6)}$. Human JAK family consists of four JAKs: JAK1, JAK2, JAK3 and TYK2. Each JAK member comprises numerous different domains as follows: $\mathrm{N}$-terminal FERM domain which is responsible for protein-protein interactions, as adaptor and scaffolding interactions with membrane associated proteins $(7,8)$ (Fig. 1). The $\mathrm{SH}_{2}$ (Src homology 2) domain is a motif containing approximately 100 residues that binds to phosphotyrosine residues and lead to the activation and dimerization of STATs ${ }^{(9)}$. Central pseudokinase domain is homology to Protein Tyrosine Kinases (PTK) domain, lacks of catalytic function and appears to have a regulatory role ${ }^{(10)}$. PTK domain is located at the C-terminus and is responsible for phosphorylation of specific tyrosine residues positioned on special downstream substrates ${ }^{(11)}$. The human STAT family contains seven STATs: STAT1, 2, 3, 4, 5A, 5B and 6.

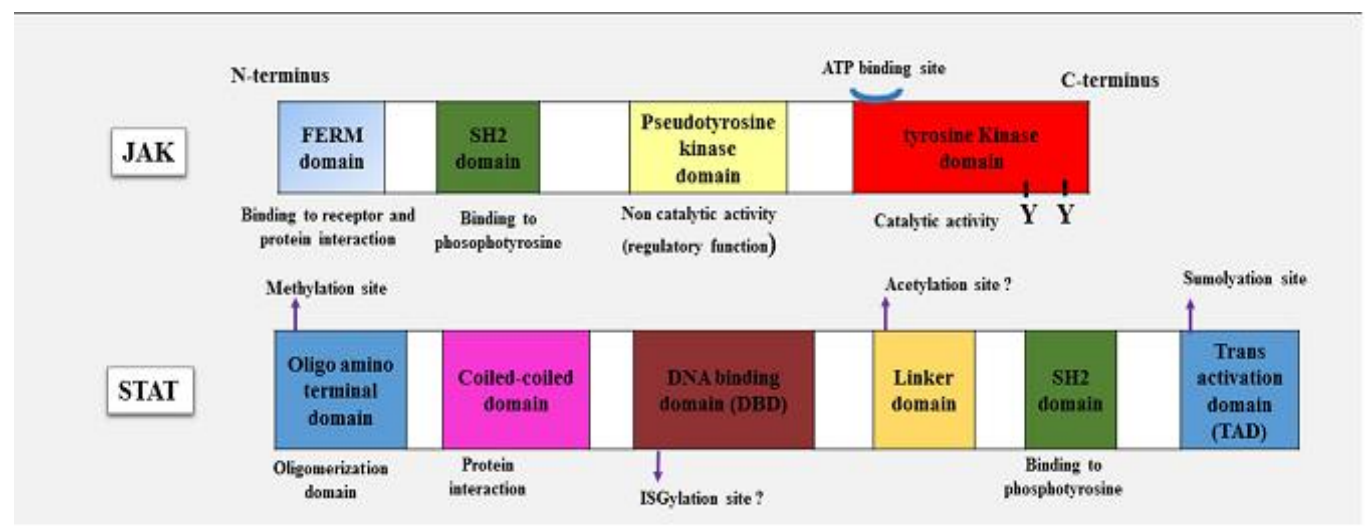

Figure 1: The structure of the JAK-STAT domains and its functions ${ }^{(8)}$

Unique $\mathrm{N}$ terminus region involves in STAT regulation, coiled-coil domain, which is involved in protein-protein interactions and nuclear export. The DNA-binding domain contains a $S$ type immunoglobulin fold. It facilitates sequence-specific binding. C-terminus region is also called Trans-Activation Domain (TAD) and contains a highly conserved tyrosine residue. JAKs are activated upon cytokine attachment and intracellu- lar region of the receptor is phosphorylated as a docking site for STATs to be recruited and phosphorylated ${ }^{(12)}$ (Figure 1). STATs start hetero- or homo-dimerization through $\mathrm{SH}_{2}$ domains. STATs were Phosphorylated and translocated to the nucleus in import in $a-5$ dependent manner. Then, dimerized STATs attach to specific DNA sequences to regulate transcription of their own target genes ${ }^{(6)}$ (Figure 2). 


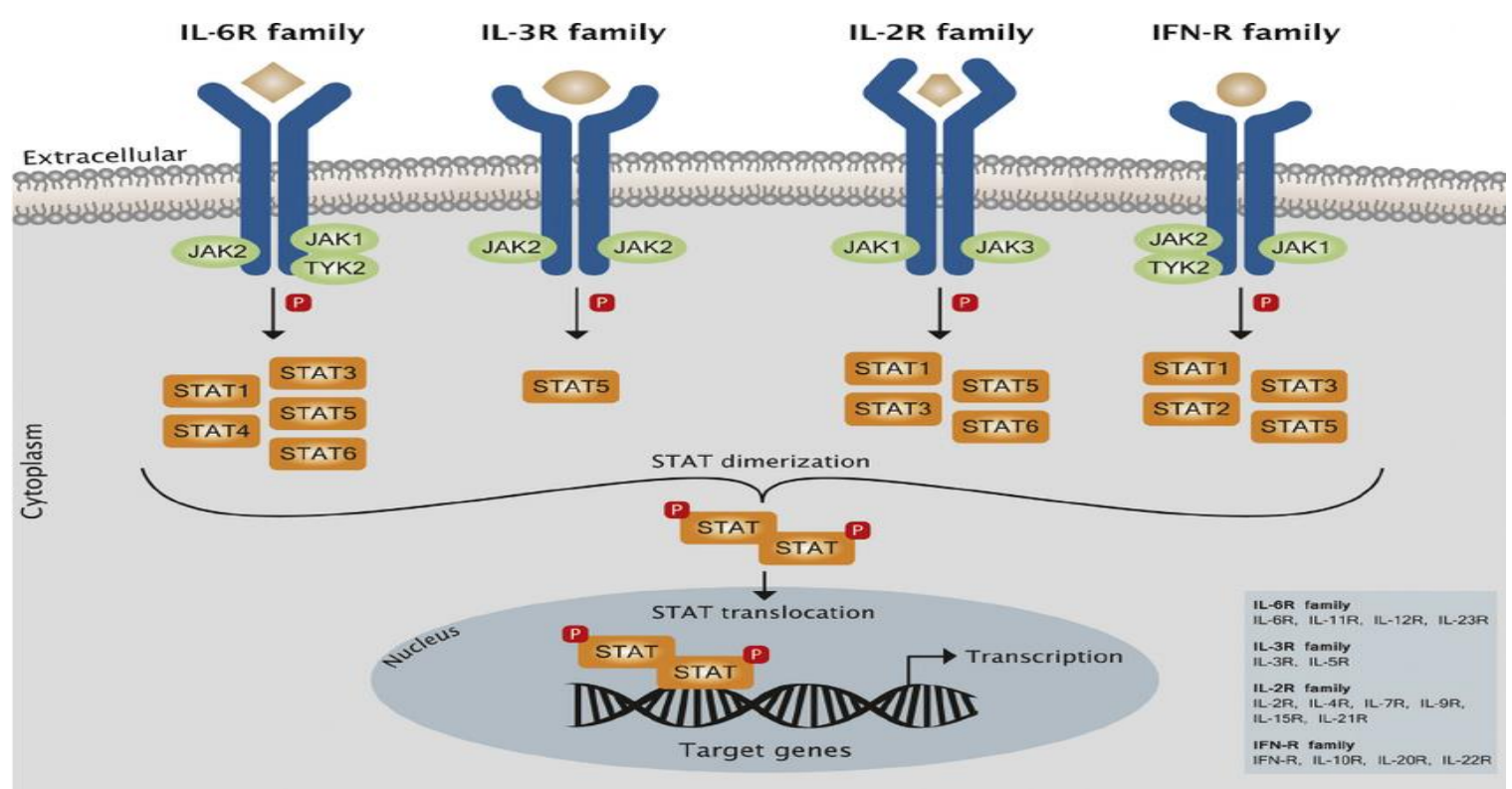

Figure 2: The JAK/STAT signaling pathway.

Cytokines exert their biologic effects through binding to specific receptors on the cell surface ${ }^{(6)}$

\section{Inhibitors of the JAK-STAT pathway}

Suppressors of Cytokine Signaling (SOCSs): SOCS protein family are consisted of eight members, including SOCS1, SOCS2, SOCS3, SOCS4, SOCS5, SOCS6, SOCS7 and cytokine-inducible $\mathrm{SH} 2$ domain protein (CIS or $\mathrm{CISH})^{(13)}$. There was found that they play an essential role in immune regulation ${ }^{(14)}$. Each SOCS proteins contain an approximately 40 amino acid boxes, called SOCS box, and a central $\mathrm{SH}_{2}$ domain. $\mathrm{SH} 2$ domain directly binds to phosphorylated tyrosines of activated JAKs; therefore, both recruitment of signal transducer adaptors such as STATs and kinase activity of JAKs is blocked. Also, $\mathrm{SH}_{2}$ determines the target of degradation. SOCS proteins preferentially regulate the termination of JAK-STAT signaling process( ${ }^{(15)}$.

Protein Inhibitors of Activated STAT (PIASs): The mammalian PIAS protein family contains 4 members, including PIAS1 (Gu binding Protein), PIAS2 (PIASx),PIAS3, and PIAS4 (PIASy) ${ }^{(16)}$. The PIAS proteins combined to dimers of activated STATs (not monomer STAT) and prohibit them from attached to specific sequences in $\mathrm{DNA}^{(6)}$.
Upon cytokine signaling, PIAS1, PIAS3, PIASX and PIASy interact with STAT1, STAT3, STAT4 and STAT1, respectively. PIAS1 and PIAS3 inhibit the DNA binding capability of STAT1 and STAT3, respectively while PIASX and PIASY slow down STAT4- and STAT1dependent gene transcription while they do not modify the DNA binding capability of STAT4 and STAT1 ${ }^{(17) .}$

Protein tyrosine phosphatases (PTPs): PTPs are the third protein groups which negatively regulate the JAK-STAT pathway functions. They are dephosphorylate tyrosine residues included in signaling pathways, then reverse JAK-STAT activity. PTPs are CD45, SHP1and SHP2 ${ }^{(18)}$.

\section{Therapeutic targets of JAK/STAT pathway}

Many of immunological disorders have been happened through different cytokine receptors throughout the JAK-STAT pathway, particularly $T$ cell mediated diseases. Thus, targeting of this pathway has gained huge attraction ${ }^{(19)}$. New drugs were introduced to inhibit JAK and STAT molecules. Although only few JAK inhibitors (JAKinib) are FDA approved, other JAKinibs and possible STAT inhibitors are being developed 
by passing preclinical evaluations and clinical trials ${ }^{(20)}$, (Table 1). Ruxolitinib is the first FDA-approved JAK inhibitor (JAKi), which is a JAK $1 / 2$ inhibitor, was developed for the treatment of myeloproliferative disorders associated with $\mathrm{JAK}_{2}{ }^{(21)}$. The 2 nd drug, tofacitinib, which is a JAK $1 / 3$ inhibitor, was developed for the treatment of patients with rheumatoid arthritis ${ }^{(22)}$. Both are under investigation (Table 2). A recent immunohistochemical study demonstrated a prominent expression of activated JAK and STAT members in ISD including PSO, AD and $\mathrm{LE}^{(23)}$.

Table 1: a number of Jak inhibitors and STAT inhibitors ${ }^{(8)}$

\begin{tabular}{|c|c|c|c|}
\hline Drug & Targt & Disease & Status \\
\hline Ruxolitinib (INC424) & JAK1, JAK2 & $\begin{array}{l}\text { Polycythemia, Psoriasis (topical), myelofibrosis, } \\
\text { Various cancers, }\end{array}$ & FDA approved \\
\hline Tofacitinib & $\begin{array}{l}J_{A K}>J_{A} A K_{1}> \\
>(J A K 2)\end{array}$ & $\begin{array}{l}\text { RA, Psoriasis, Spondyloarthropathy, Transplant re- } \\
\text { jection, ulcerative colitis }\end{array}$ & FDA approved \\
\hline Oclacitinib & JAK1 & Canine allergic dermatitis & FDA approved \\
\hline Baricitinib & JAK1, JAK2 & $\begin{array}{l}\text { RA Psoriasis, diabetic nephropathy, SLE, Atopic } \\
\text { dermatitis }\end{array}$ & Phase III Phase II \\
\hline Momelitinib & JAK1, JAK2 & Myelofibrosis & Phase III \\
\hline Peficitinib & $\mathrm{JAK}_{1}, \mathrm{JAK}_{3}$ & RA Psoriasis & Phase III Phase II \\
\hline INCB039110 & JAK1, JAK2 & Psoriasis, RA & Phase II \\
\hline AZD1480 & JAK1, JAK2 & Myeloproliferative diseases, various cancers & Phase I \\
\hline ISIS-STAT3Rx (AZD9150) & STAT3 & Various cancers & Phase II \\
\hline OPB-31121 & STAT3 & Various cancers & Phase I \\
\hline
\end{tabular}

Table 2: JAK/STAT inhibitors in clinical development for inflammatory skin diseases ${ }^{(24)}$

\begin{tabular}{lllll}
\hline ISD & Inhibitor & Target & Drug administration & Stage of development \\
\hline AA & PF-06700841 & TYK2/ & Oral & Phase 2 \\
& PF-06651600 & JAK3 & Oral & Phase 2 \\
& Ruxolitinib & JAK1/JAK2 & Topical formulation & Phase 2 \\
& Tofacitinib & JAK1/JAK3 & Topical formulation & Phase 2 \\
& Tofacitinib & JAK1/JAK3 & Oral & Phase 2 \\
AD & ABT-494 & JAK1 & Oral & Phase 2 \\
& Baricitinib & JAK1/JAK2 & Oral & Phase 2 \\
& PF-04965842 & JAK1 & Oral & Phase 2 \\
& Tofacitinib & JAK1/JAK3 & Topical formulation & Phase 2 \\
GvHD & Baricitinib & JAK1/JAK2 & Oral & Phase 2 \\
& Ruxolitinib & Ruxolitinib & Oral & Phase 3 \\
LE & Baricitinib & JAK1/JAK2 & Oral & Phase 2 \\
& Tofacitinib & JAK1/JAK3 & Oral & Phase 1 \\
& GSK2586184 & JAK1 & Oral & Phase 1 \\
PSO & Baricitinib & JAK1/JAK2 & Oral & Phase 2 \\
& GSK2586184 & JAK1 & Oral & Phase 2 \\
& PF-04965842 & JAK1 & Oral & Phase 2 \\
& PF-06263276 & Pan-JAK & Topical formulation & Phase 1 \\
& Ruxolitinib & JAK1/JAK2 & Topical formulation & Phase 2 \\
& STA-21 & STAT3 & Topical formulation & Phase 2 \\
& Tofacitinib & JAK1/JAK3 & Oral & Phase 3 \\
& Tofacitinib & JAK1/JAK3 & Eye drops & Phase 2 \\
& Tofacitinib & JAK1/JAK3 & Ophthalmic emulsion & Phase 2 \\
\hline
\end{tabular}

AA: alopecia areata; AD: atopic dermatitis; GvHD: graft-versus-host disease; LE: lupus erythematous; PSO: psoriasis; SJS: Sjogren's syndrome. www.clinicaltrials.gov

\section{Alopecia areata}

Alopecia areata (AA) is an ISD characterized by sudden onset of hair loss in circular areas of the scalp or body. Patients usually have a family history of atopy , AA ,or other autoimmune disorders ${ }^{(25)}$. At the cellular level, the hair follicles appear to lose their immune-privileged status and are attacked by both autoreactive CD8+ T cells and NK T cells (Figure. 3A). 
$\mathrm{CD} 8+\mathrm{NKG} 2 \mathrm{D}+\mathrm{T}$ cells activity and their IFN$\gamma$ secretion are enhanced by IL-2 and IL-15 signaling. While these type I/II receptor dependent cytokines are so important in AA, interfering with the JAK/STAT pathway may be of therapeutic benefit. in addition, AA hair follicles of patients and experimental mice both show phosphorylation of STAT proteins including both STAT1 and STAT3. These lead to inhibition of the signaling proteins of IFN- $\gamma$ (STAT1), IL-2 (STAT5) and IL-15 (STAT5) (26). An important and effective way to attenuate AA by simultaneous downstream of IFN- $\gamma$, IL- 2 and IL-15 signaling is the utilize of a JAKi that acts upstream of STATs. The oral JAK1/JAK2 inhibitors ruxolitinib and baricitinib both seem to induce hair-regrowth in patients with $A A^{(27)}$. Similarly, oral tofacitinib may induce hair-regrowth in a $A A$ patients in retrospective studies $(28,29)$. Now, the two JAKi, tofacitinib and ruxolitinib, are in phase 2 trials for topical treatment of AA. Besides tofacitinib, two other compounds - PF-06700841 and PF06651600 - are under investigation as systemic drugs for AA in phase 2 trials (Table $2)^{(24,30,31)}$.

\section{Atopic dermatitis}

Atopic dermatitis (AD) is a chronic inflammatory skin disease. It is characterized by pruritus and eczematous lesions. The interaction of skin DCs with irritants with pathogens and allergens were occurred due to the impaired epidermal barrier. The cytokine in $A D$ is dominated by the Th2 cytokines IL-4, IL-13 and IL-33, which down regulate Th17/IL-23 responses, and the creation of antimicrobial peptides. The Th2 response favors an inflammation relating with type 2 ILCs, eosinophils, mast cells and itch-promoting cytokines like IL-31 ${ }^{(32)}$ (Fig. 3B). Since dupilumab will be the first biologic to be approved for atopic dermatitis, anti-cytokine treatments targeting IL-22 or IL-31 are in early developmental phase 1/2 studies(33). The significance role of multiple type I/II cytokine receptor-using mediators, as IL-4, IL-13, IL-22, IL-31 and IFN- $\gamma$, in $A D$ pathogenesis suggest that interfering with the JAK/STAT pathway could be a more potent therapeutic approach than neutralization of a single cytokine ${ }^{(34)}$. Currently, many JAKi are under investigation for the treatment of human AD (Table 2). For example, the oral JAK1/JAK2 inhibitor baricitinib and the JAK1 inhibitors ABT-494 and PF-04965842 are all in phase 2 studies (www.clinicaltrials.gov). Topical JAKi are also in development for the treatment of $A D^{(24,35)}$. In $A D$, a $2 \%$ tofacitinib ointment has showed a major enhancement of the EASI of $>80 \%$ compared to $30 \%$ improvement in the vehicle group ${ }^{(36)}$. This enhancement was related with a decrease in pruritus.

\section{Psoriasis}

Psoriasis is a chronic ISD with hereditary character that can be aggravated by endogenous or exogenous triggering factors ${ }^{(37)}$. These genetic factors were studied and reported a group of genes encoding cytokine and cytokine receptors: IL23A, IL12B, IL23R, IL4/IL13, together with their downstream signaling molecules: TYK2 and STAT3. The pathophysiology process of psoriasis is occurred mainly by an IL-17A+ Th17 immune response. Several cytokines that are very much expressed in psoriatic skin lesions signal through the JAK/STAT pathway, including IL-19, IL-20, IL-22 and IL23 (Figure $3 \mathrm{C}$ ) ${ }^{(38)}$. There was reported an active STAT3 is typically present both in psoriatic immune cells and in psoriatic keratinocytes produce epidermal hyperplasia. IL-23 is mostly produced by DCs and stimulation of the IL-23R in T cells leads to recruitment of JAK2/TYK2 and downstream to the activation of STAT3. STAT3 is significant for regulating the expression of IL-23R and for the expression of IL-17A, IL${ }_{17} \mathrm{~F}$ and IL-22 in T cells ${ }^{(39)}$. The tofacitinib, 
JAKi, slow down IL-23R up-regulation in T cells which has been stimulated with IL-6 and IL-23. Furthermore, tofacitinib slow down the development of IL-23-dependent Th17 cells and the expression of IL-17A, IL$17 \mathrm{~F}, \mathrm{IL}-21$ and IL-22. This inhibitory activity is of significance, since IL-23-dependent Th17 cells play a crucial role in autoimmune diseases. In humans, biologics that neutralize IL-17A or IL-23 have been shown to be highly effective in the treatment of psoriasis ${ }^{(40)}$. Recently, multiple JAKi are tested for the topical or systemic treatment of psoriasis (Table 2). The tofacitinib, (JAK1/ $\mathrm{JAK}_{3}$ inhibitor) showed an improvement of $>75 \%$ of the psoriasis area and severity index (PASI-75) in 40-64\% of patients at week 12 of treatment in a phase 3 trial (41). The JAK1/JAK2 inhibitor baricitinib achieved a PASI-75 response in $43-54 \%$ of patients at week 12 in a phase 2 trial. Other oral JAK inhibitors, like the JAK1 inhibitors GSK2586184 or PF-04965842, are in early phase 2 development for psoriasis. Two JAK inhibitors, the JAK1/JAK2 inhibitor ruxolitinib and the pan-JAK inhibitor PF-06263276 were developed as topical formulation for psoriasis and are currently tested in phase 2 and phase 1 , respectively (Table 2$)^{(24)}$.

\section{Lupus Erythematosus}

Lupus erythematous (LE) is an inflammatory autoimmune disease, characterized by red discoid or anular skin lesions, circulating autoantibodies and, in patients with systemic disease, deposition of immune complexes in various tissues(42). LE Patients have showed diverse sensitivities to IFN signaling due to mutation or genetic STAT4 variants in the STAT4 gene locus. Also, STAT3 signaling is significant in LE pathogenesis as LE demonstrate impaired T follicular helper (TFH) cell responses, germinal center $B$ cells, autoantibody production and nephritis (Fig. 3D). IL-12-induced STAT4 activation and IL-6-induced STAT3 activation both require JAK2 signaling(43).
The tofacitinib (JAK1/JAK3 inhibitor) and JAK2 inhibitor CEP-33779 could be reduced autoantibody levels and improve lupus nephritis(44). Furthermore, tofacitinib also can improve lupus-like skin inflammation. Three oral JAKi are now under evaluation for the treatment of patients with systemic LE: baricitinib, tofacitinib and the JAK1 inhibitor GSK2586184 (Table 2)(24).

\section{Sjogren's syndrome}

Sjogren's syndrome (SJS), dry eye syndrome, is a chronic inflammation in exocrine glands resulting in growing dryness of mucous membranes. One member of the nuclear IkB family of proteins, IkB- $\zeta$ has a central role in the pathogenesis of SJS. The experimental mice with deletion of IkB- $\zeta$ or its transcriptional regulator STAT3 in epithelial tissues, i.e. the lacrimal glands develop SJS-like autoimmune disease. STAT3 and IKB- $\zeta$ have several roles in epithelial cells than in lymphoid cells (Fig. $3 \mathrm{E}$ ). While the deletion of these factors in epithelial cells of the lacrimal glands induces SJS-like disease, STAT3 and IKB- $\zeta$ expression in T cells is important for Th17 cell development. Yet, the inhibition of the JAK/STAT pathway seems to be beneficial in patients with SJS(45). The tofacitinib, $\mathrm{JAKi}$, that has been tested in phase 2 randomized controlled trials of dry eye disease with ophthalmic emulsions or eye drops (Table 2) (24). JAK1/3 inhibition improved signs and symptoms of dry eye, decreased HLA-DR conjunctival cell surface expression and also the expression levels of inflammatory cytokines in the tears of SJS patients $(46,47)$.

\section{Future Directions}

Currently, there are many clinical trials on STAT inhibitors for ISDs and they are in early development while JAK inhibitor programs are already in advanced stages of clinical development ${ }^{(48)}$. Several strategies were done to select one inhibitor. 

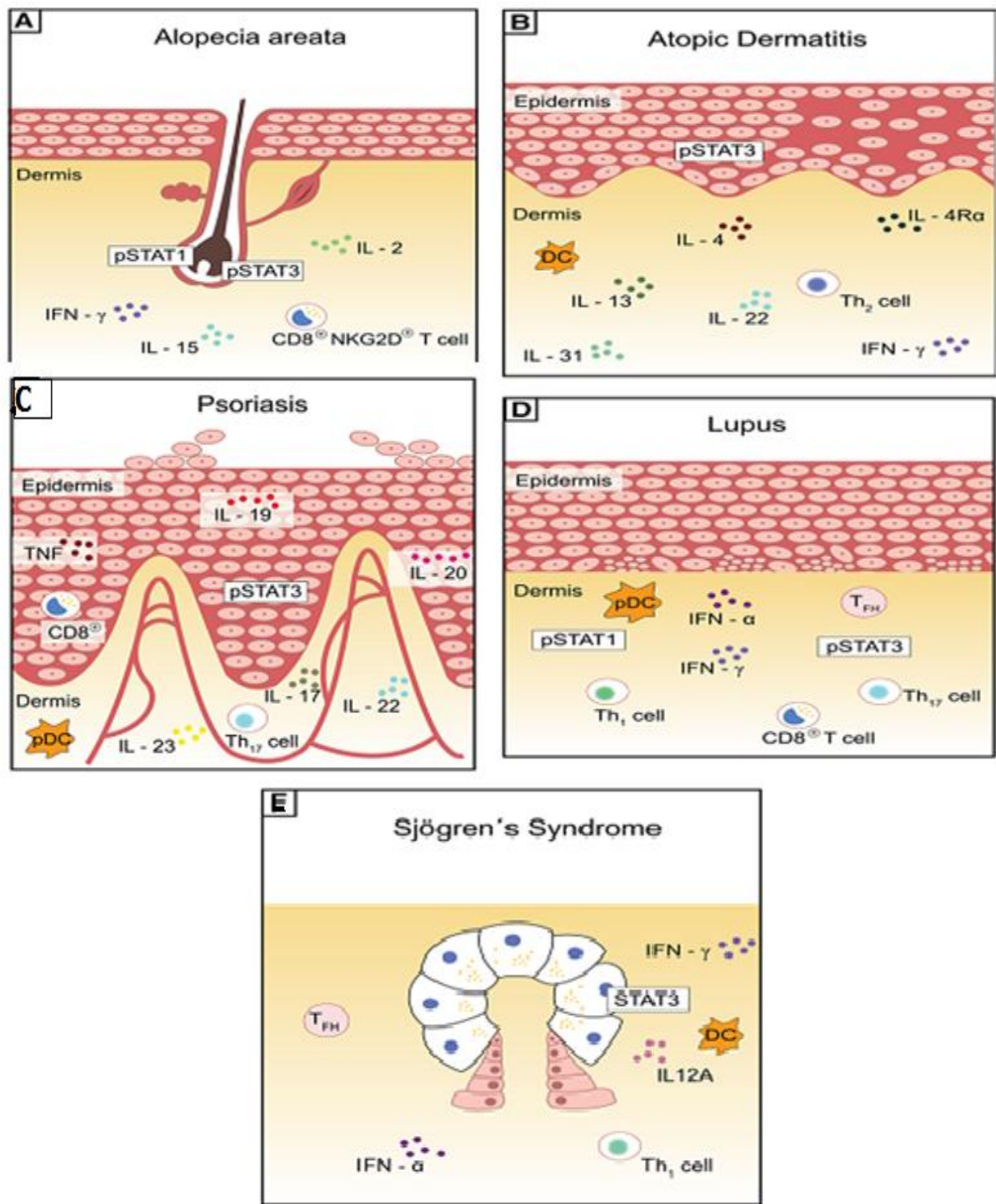

Figure 3: pathophysiology of ISDs with T-cell subsets and associated cytokines that signal through the JAK/STAT pathway. (A) The hair follicles in AA are under attack by a cytotoxic NK T cell response enhanced by the cytokines IL-2, IL-15 and IFN- $\gamma$. (B) AD is initiated by an IL-4+ and IL-13+ Th2 response, but also other cytokines such as IL-22, IL-31 and IFN- $\gamma$ are concerned in the chronic disease phase. (C) PSO is a Th17 disease with high appearance of IL-17, TNF and Th17-associated cytokines like IL-19, IL-22 and IL-23. (D) Th1, Th17 and TFH cells are the main T cell subsets in the pathogenesis of LE with a high up IFN signature. (E) In SJS the inflammation of exocrine glands is mediated by an IFN-dominated Th1/TFH immune response with STAT3 having different roles in epithelial and immune cells. DC: dendritic cell; pDC: plasmacytoid dendritic cells producing type I IFN; Th: T helper cell; TFH: follicular T helper cell(24). 
They tried to inhibit STAT3 only and to a lesser extent STAT5. These have integrated anti-sense, small molecule inhibitors and decoy oligonucleotides. The mainly capable of these compounds is the STAT3 inhibitor STA-21. This molecule could inhibit STAT3 DNA binding activity and STAT3 dimerization without affecting STAT3 phosphorylation ${ }^{(49)}$. Furthermore, JAKi has high risk of infection and malignancies because JAKi inhibits multiple JAKs in the first generation. Thus, the need for a highly selective JAKi is an important issue ${ }^{(50)}$. Currently, the selective JAKi that targets individual JAKs is in development in the second generation JAKi ${ }^{(51)}$. Further future clinical trials will be done to show the comparable efficacy of the first generation JAKi and the second generation JAKi, and which have more acceptable toxicities. In ISDs, JAKi could enhance the treatment modalities. The mainly treatment advantage with JAKi is their topical administration to replace topical glucocorticosteroid as they have many cutaneous side effects. The pan-JAKi topical treatment is already in phase $1^{(24)}$.

\section{Conclusion}

Currently, the small molecular inhibitors which Interfering with the JAK/STAT signaling cascade are used not only in the malignant disorders but also in the autoimmune disease. JAKi can enhance or inhibit many immunological processes as they interfere with innate immune responses, Thcell differentiation, B-cell activation and antibody production. Consequently, ISDs could be a perfect group of diseases to test such inhibitors, since the type I/II cytokine receptor-using cytokines are significantly drawn in ISDs.the first generation which targeting multiple JAK/STAT-associated cytokines at the same time as the second generation which targeting the signaling pathway itself is an alternative approach to neutralizing single cytokines by antibodies.

\section{References}

1. Dainichi $T$, Hanakawa $S$, Kabashima K.Classification of inflammatory skin diseases: a proposal based on the disorders of the three-layered defense systems, barrier, innate immunity and acquired immunity. J Dermatol Sci 2014; 76:81-9.

2. Ghoreschi K, Laurence A, O'Shea JJ.Janus kinases in immune cell signaling. Immunol Rev 2009; 228:273-87.

3. Renauld JC.Class II cytokine receptors and their ligands: key antiviral and inflammatory modulators. Nat Rev Immunol 2003;3:667-76.

4. Liongue C, O'Sullivan LA, Trengove MC, Ward AC. Evolution of JAK-STAT pathway components: mechanisms and role in immune system development. PloS one 2012; 7:e32777.

5. Stark GR, Darnell JE, Jr.The JAK-STAT pathway at twenty. Immunity 2012; 36:503-14.

6. Rawlings JS, Rosler KM, Harrison DA.The JAK/STAT signaling pathway. J Cell Sci 2004; 117:1281-3.

7. Tepass U.FERM proteins in animal morphogenesis. Curr Opin Genet Dev 2009; 19:357-67.

8. Seif $F$, Khoshmirsafa $M$, Aazami $H$, et al.The role of JAK-STAT signaling pathway and its regulators in the fate of T helper cells. Cell Commun Signal : CCS 2017; 15:23.

9. Liu BA, Jablonowski K, Raina $\mathrm{M}$, et al.The human and mouse complement of SH2 domain proteins-establishing the boundaries of phosphotyrosine signaling. Mol Cell 2006; 22:851-68.

10. Kisseleva T, Bhattacharya $S$, Braunstein $J$, Schindler CW.Signaling through the JAK/STAT pathway, recent advances and future challenges. Gene 2002; 285:1-24.

11. Lim WA, Pawson T.Phosphotyrosine signaling: evolving a new cellular 
communication system. Cell 2010; 142:661-7.

12. Shuai K, Liu B.Regulation of JAK-STAT signalling in the immune system. Nat Rev Immunol 2003; 3:900-11.

13. Alexander WS.Suppressors of cytokine signalling (SOCS) in the immune system. Nat Rev Immunol 2002; 2:410-6.

14. Palmer DC, Guittard GC, Franco Z, et al.Cish actively silences TCR signaling in CD8+ $T$ cells to maintain tumor tolerance. J Exp Med 2015; 212: 2095113.

15. Kamizono S, Hanada $\mathrm{T}$, Yasukawa $\mathrm{H}$, et al.The SOCS box of SOCS-1 accelerates ubiquitin-dependent proteolysis of TELJAK2. J Biol Chem 2001; 276:12530-8.

16. Lao M, Shi M, Zou $Y$, et al.Protein Inhibitor of Activated STAT3 Regulates Migration, Invasion, and Activation of Fibroblast-like Synoviocytes in Rheumatoid Arthritis. J Immunol 2016; 196:596-606.

17. Arora $\mathrm{T}$, Liu B, He $\mathrm{H}$, et al.PIASx is a transcriptional co-repressor of signal transducer and activator of transcription 4. J Biol Chem 2003; 278:2132730.

18. Penninger JM, Irie-Sasaki J, Sasaki T, Oliveira-dos-Santos AJ.CD45: new jobs for an old acquaintance. Nat Immunol 2001; 2:389-96.

19. O'Shea JJ, Schwartz DM, Villarino AV, et al.The JAK-STAT pathway: impact on human disease and therapeutic intervention. Ann Rev Med 2015; 66:311-28.

20. Ghoreschi K, Jesson MI, Li X, et al.Modulation of innate and adaptive immune responses by tofacitinib (CP690,550). J Immunol 2011; 186:4234-43.

21. Verstovsek S, Kantarjian H, Mesa RA, et al.Safety and efficacy of INCB018424, a JAK1 and JAK2 inhibitor, in myelofibrosis. N Engl J Med 2010; 363:111727.

22. Fleischmann R, Kremer J, Cush J, et al.Placebo-controlled trial of tofacitinib monotherapy in rheumatoid arthritis. $\mathrm{N}$ Engl J Med 2012;367:495-507.

23. Alves de Medeiros AK, Speeckaert R, Desmet $E$, et al.JAK3 as an Emerging
Target for Topical Treatment of Inflammatory Skin Diseases. PloS one 2016; 11:e0164080.

24. Welsch K, Holstein J, Laurence A, Ghoreschi K.Targeting JAK/STAT signalling in inflammatory skin diseases with small molecule inhibitors. Eur J Immunol 2017; 47:1096-107.

25. Gilhar A, Etzioni A, Paus R.Alopecia areata. N Engl J Med 2012; 366:1515-25.

26. Xing L, Dai Z, Jabbari A, et al.Alopecia areata is driven by cytotoxic $T$ lymphocytes and is reversed by JAK inhibition. Nat Med 2014; 20:1043-9.

27. Mackay-Wiggan J, Jabbari A, Nguyen N, et al.Oral ruxolitinib induces hair regrowth in patients with moderate-tosevere alopecia areata. JCl insight 2016; 1:e89790.

28. Craiglow BG, Liu LY, King BA.Tofacitinib for the treatment of alopecia areata and variants in adolescents. J Am Acad Dermatol 2017; 76:29-32.

29. Liu LY, Craiglow BG, Dai F, King BA.Tofacitinib for the treatment of severe alopecia areata and variants: A study of 90 patients. J Am Acad Dermatol 2017; 76:22-8.

30. Liu LY, King BA.Tofacitinib for the Treatment of Severe Alopecia Areata in Adults and Adolescents. J Invest Derm Symp P 2018; 19:S18-s20.

31. Liu LY, Craiglow BG, King BA.Tofacitinib $2 \%$ ointment, a topical Janus kinase inhibitor, for the treatment of alopecia areata: A pilot study of 10 patients. J Am Acad Dermatol 2018; 78:403-4.e1.

32. Werfel T, Allam JP, Biedermann T, et al.Cellular and molecular immunologic mechanisms in patients with atopic dermatitis. J allergy clin immunol 2016; 138:336-49.

33. Mennini M, Dahdah L, Fiocchi A.Two Phase 3 Trials of Dupilumab versus Placebo in Atopic Dermatitis. N Engl J Med 2017; 376:1090.

34. Sehra S, Krishnamurthy $P$, Koh B, et al.Increased Th2 activity and diminished skin barrier function cooperate in allergic skin inflammation. Eur J Immunol 2016; 46:2609-13. 
35. Bissonnette R.JAK inhibitors appear to have a bright future in the treatment of atopic dermatitis. Br J Dermatol 2018; 178:321.

36. Bissonnette $R$, Papp KA, Poulin $Y$, et al.Topical tofacitinib for atopic dermatitis: a phase lla randomized trial. $\mathrm{Br} \mathrm{J}$ Dermatol 2016; 175:902-11.

37. Greb JE, Goldminz AM, Elder JT, et al.Psoriasis. Nat Rev Dis primers 2016; 2:16082.

38. Cochez PM, Michiels C, Hendrickx E, et al.AhR modulates the IL-22-producing cell proliferation/ recruitment in imiquimod-induced psoriasis mouse model. Eur J Immunol 2016; 46:1449-59.

39. Ghoreschi K, Laurence A, Yang XP, et al.Generation of pathogenic $T(H) 17$ cells in the absence of TGF-beta signalling. Nature 2010; 467:967-71.

40. Kopp T, Riedl E, Bangert C, et al.Clinical improvement in psoriasis with specific targeting of interleukin-23. Nature 2015; 521:222-6.

41. Papp KA, Menter MA, Raman M, et al.A randomized phase $2 \mathrm{~b}$ trial of baricitinib, an oral Janus kinase (JAK) 1/JAK2 inhibitor, in patients with moderate-tosevere psoriasis. $\mathrm{Br} J$ Dermatol 2016; 174:1266-76.

42. Dorgham K, Amoura Z, Parizot C, et al.Ultraviolet light converts propranolol, a nonselective beta-blocker and potential lupus-inducing drug, into a proinflammatory AhR ligand. Eur J Immunol 2015; 45:3174-87.

43. Ding $C$, Chen $X$, Dascani $P$, et al.STAT3 Signaling in B Cells Is Critical for Germinal Center Maintenance and Contributes to the Pathogenesis of Murine Models of Lupus. I Immunol 2016; 196:4477-86.

44. Furumoto Y, Smith CK, Blanco L, et al.Tofacitinib Ameliorates Murine Lupus and Its Associated Vascular Dysfunc- tion. Arthritis \& Rheumatology (Hoboken, NJ) 2017; 69:148-60.

45. Johansen $C$, Mose $M$, Ommen $P$, et al.IkappaBzeta is a key driver in the development of psoriasis. Proc Natl Acad Sci U S A 2015; 112:E5825-33.

46. Liew SH, Nichols KK, Klamerus KJ, et al.Tofacitinib (CP-690,550), a Janus kinase inhibitor for dry eye disease: results from a phase $1 / 2$ trial. Ophthalmology 2012; 119:1328-35.

47. Del Papa N, Vitali C.Management of primary Sjögren's syndrome: recent developments and new classification criteria. Ther Adv Musculoskelet Dis 2018; 10:39-54.

48. Furqan $M$, Akinleye $A$, Mukhi $N$, et al.STAT inhibitors for cancer therapy. J Hematol Oncol 2013; 6:90.

49. Miyoshi K, Takaishi M, Nakajima K, et al.Stat3 as a therapeutic target for the treatment of psoriasis: a clinical feasibility study with STA-21, a Stat3 inhibitor. J Invest Dermatol 2011; 131: 108-17.

50. Curtis JR, Xie F, Yun H, Bernatsky S, Winthrop KL.Real-world comparative risks of herpes virus infections in tofacitinib and biologic-treated patients with rheumatoid arthritis. Ann Rheum Dis 2016; 75:1843-7.

51. Forster M, Chaikuad A, Bauer SM, et al.Selective JAK3 Inhibitors with a Covalent Reversible Binding Mode Targeting a New Induced Fit Binding Pocket. Cell Chem Biol 2016; 23:133540.

Author: Dr. Hanan Omar Assistant Prof. Clinical Pathology Faculty of Medicine, Suez-Canal University, Ismailia, Egypt Email: hananhassan1978@gmail.com Phone: + 201093627403 